\title{
THE CIRCUITRY AND FUNCTIONAL ROLE OF INHIBITORY PROJECTIONS TO NEURONS SENSITIVE TO INTERAURAL INTENSITY DIFFERENCE IN THE INFERIOR COLLICULUS
}

\author{
T.J. PARK, G.D. POLLAK, J.A. WINER* and D.T. LARUE* \\ Department of Zoology, University of Texas, Austin, Texas 78712, USA \\ *Division of Neurobiology, Department of Molecular and Cell Biology, University of California, \\ Berkeley, California 94720-2097, USA
}

\begin{abstract}
Neurons sensitive to interaural intensity difference were examined in the inferior colliculus of the mustache bat. The inferior colliculus receives a convergence of inputs from the lower auditory nuclei, some excitatory and some inhibitory. The origins of the inhibitory projections to neurons sensitive to interaural intensity difference were identified by labeling projection cells with a retrograde tracer, then using antibodies for GABA and glycine on the same neurons. The physiological effects of these transmitters were assessed on single neurons by applying the transmitters and their blockers while acoustically stimulating the cells. For some cells, binaural characteristics were sharpened by inhibitory transmitters while others were unaffected. For still others, inhibition appears to fully create their binaural characteristics within the colliculus.

Les neurons sensitifs à la différence d'intensité interaural ont été examinés dans le colliculus inférieur de la chauve-sourie moustachue (Pteronotus parnellii). Le colliculus inférieur reçoit une convergence de signeaux des centres auditifs inférieurs, certains excitant, certains inhibitant. Les origines des projections inhibitantes des neurons sensitifs aux différences d'intensité interaural ont été identifié par cellules co-labellantes pour un tracer rétrograde et pour anticorps contre les transmitteurs inhibitants GABA et glycine. Les effets de ces transmitteurs ont été évalués sur les neurons individuels en applicant les transmitteurs et leurs antagonistes tout en stimulant accoustiquement les cellules. Les charactéristiques binaurales ont été accentuées pour certains cellules par des transmitteurs inhibitant tandis que d'autres cellules restaient inchangés. Cependant, d'autres cellules apparemment nécessitent les signeaux inhibitant pour créer des charactéristiques binaural dans le colliculus inférieur.
\end{abstract}

\section{Introduction}

Virtually all animals that hear sound can localize its source and, for most animals, this ability is crucial for survival $(1,2)$. Vertebrates, including humans, rely heavily on binaural cues for localization that involves differences in the sound's waveform between the ears (3). One such cue that plays an important role in sound localization is the interaural intensity difference (IID).

Intensity differences between the ears are very small when a sound source is directly in front of an animal, but, as the sound source shifts to positions away from the front, the sound's intensity increases at the closer ear and lessens at the other ear. This is due to sound diffraction and sound shadow effects from the head. Thus, for a given frequency, the IID can indicate the position of the sound source. Localization of a stimulus using binaural difference cues is intriguing because it requires a comparison between the ears involving a very different process than localization of a visual or somatosensory stimulus, where stimulus location corresponds to a location on the sensory surface (4).

A great deal of research has been aimed at understanding how the auditory system codes spatial location from binaural intensity differences. Neurophysiological studies have shown that some auditory neurons respond to binaural stimulation in that they are excited by sound to one ear and inhibited by sound to the other. This type of cell is referred to as an excitatory/inhibitory (EI) neuron and they are present at every synaptic level of the auditory system from the superior olivary complex in the lower brainstem to the auditory cortex. EI neurons are presumed to be important for sound localization because the neuron becomes responsive as IIDs change from more intense at the inhibitory ear to more intense at the excitatory ear, and the transition point from non-responsive to responsive is relatively sharp. An individual EI 
neuron can thus indicate if a particular IID lies on one or the other side of its transition point which presumably corresponds to one or the other side of a position in space.

We have been studying excitatory/inhibitory neurons in the inferior colliculus (IC) of the mustache bat. The mustache bat is an excellent animal for this study for several reasons (5). Like all echolocating bats, this species has a relatively large and well developed auditory system indicative of the crucial role audition plays for these animals, and, like many small mammals, the mustache bat relies heavily on IIDs for localization. A unique feature of the mustache bat is that one frequency is greatly overrepresented at every level in its auditory system. This frequency corresponds to the most prominent component of the bat's echolocation call: a long, tone-like component at $60 \mathrm{kHz}$. Because the auditory regions corresponding to $60 \mathrm{kHz}$ are very large and accessible, we can identify organizational features and connections within this frequency region. Since the auditory system is tonotopically organized, our findings are representative of all frequency regions. Finally, an important feature of the mustache bat's auditory system is that it is typically mammalian in terms of cell types, nuclei, anatomical connections, and neurotransmitters.

The IC is the auditory nucleus of the midbrain. As in other mammals, the IC is a nucleus where projections from many lower nuclei converge before the auditory pathway continues to the thalamus and cortex. An examination of cell types in the large $60 \mathrm{kHz}$ region of the mustache bat's IC has revealed a large clustering of EI neurons. There is also an area of monaural units and two areas of excitatory/excitatory binaural neurons. An important finding is that each of these topologically and functionally distinct areas receives a unique pattern of innervation from the lower nuclei (6).

The extraordinary size of the $60 \mathrm{kHz}$ region of the mustache bat's IC has made possible an examination of how the EI neurons are functionally organized within one frequency region. The findings suggest that EI units act together to create patterns of activity that indicate the position of a sound source. Units located dorsally in the EI area become responsive to sound locations at the midline (small IIDs) and remain responsive to sounds contralateral to the midline, while deeper EI units become responsive to sounds located more contralateral from the midline (larger IIDs). The deeper the neuron, the more contralateral a sound location must be to induce responsiveness (7).

These findings indicate that the EI units in the IC are important for sound localization. The present study was designed to 1) identify which lower nuclei may be contributing inhibitory influences to these EI units, and 2) what functional role those inhibitory influences may play in shaping the response characteristics of the EI units.

\section{Procedure}

We first identified which of the lower nuclei send inhibitory projections to the $60 \mathrm{kHz}$ EI area of the IC. The IC was injected with a retrograde tracer to label cells projecting to the IC from lower auditory nuclei. The tracer was WGA-apoHRP-gold (WAHG), consisting of enzymatically inactivated HRP conjugated to wheat germ agglutinin and colloidal gold (8). After a 1-2 day survival period, the brains were sectioned and processed for WAHG. The sections were further processed to immunochemically identify cells containing the principal inhibitory transmitters of the auditory system, glycine and GABA. Those lower nuclei known to project to the EI area were examined for cells labeled for both WAHG and inhibitory transmitters.

We next examined how glycine and GABA affect the response properties of EI neurons in the IC. Multibarrelled glass pipettes were inserted into the IC and positioned to make extracellular recordings from individual EI neurons. One barrel was used to record neural responses to sound stimuli. The other barrels contained glycine; a glycine receptor blocker, strychnine; GABA; and a GABA receptor blocker, bicuculline. These drugs were iontophoretically ejected onto an EI neuron to assess their effects on a number of response properties including the cell's IID function.

\section{Results}

Three lower nuclei contained immunopositive cells that project to the $60 \mathrm{kHz}$ EI area of the IC. One of these, the ipsilateral lateral superior olive (LSO), contains glycinergic cells that were co-labeled with the retrograde tracer, WAHG. The other nuclei, the ipsilateral and contralateral dorsal nuclei of the lateral lemniscus (DnLL), contained GABAergic cells co-labeled with WAHG. Neurons within the IC were also identified as being GABAergic. Figure 1 is a schematic drawing of the bat brain showing the glycinergic and GABAergic projections to the EI area of the IC. 


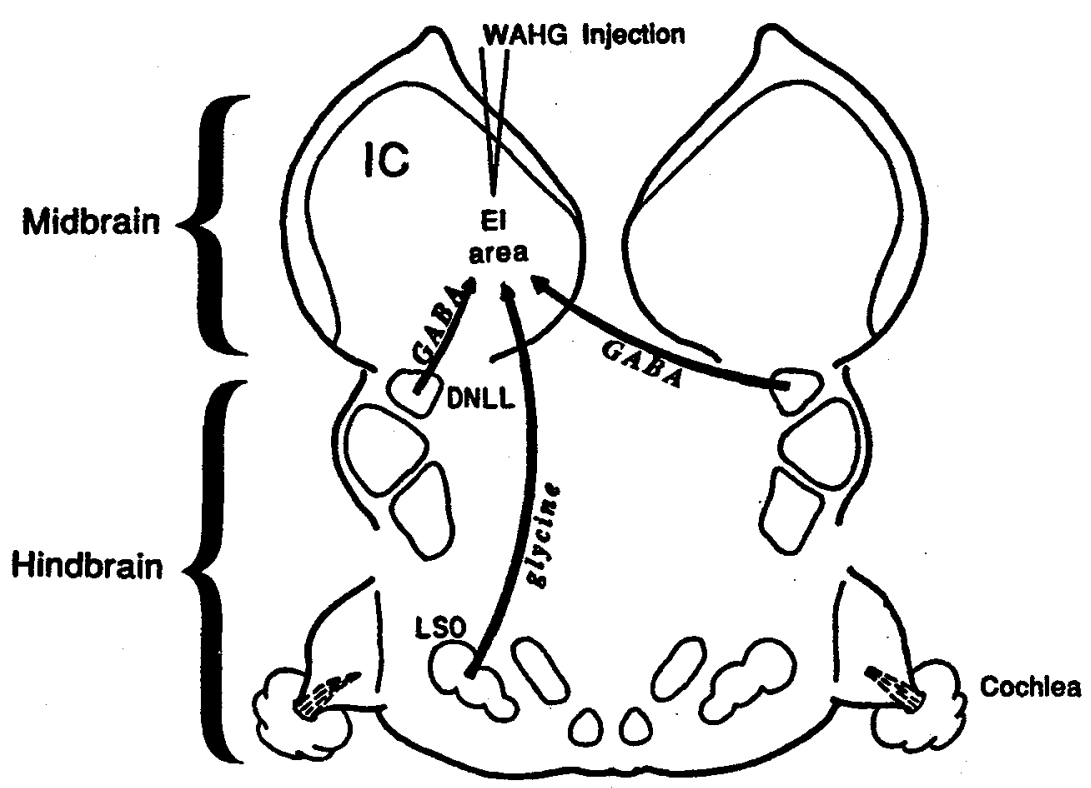

Fig. 1. - This is a schematic drawing of the mustache bat brainstem showing the inferior colliculus (IC), the nuclei of the lateral lemniscus, the nuclei of the superior olivary complex, and the cochlear nuclei. The ipsilateral lateral superior olive (LSO) sends glycinergic projections to the $60 \mathrm{kHz}$ EI area of the IC. Both of the dorsal nuclei of the lateral lemniscus (DnLL) send GABAergic projections.

The pharmacological analysis shows that all EI cells are affected in some way by both glycine and GABA. Moderate doses of either transmitter could completely silence a cell while application of blockers for either transmitter almost always increased responding. Blocking GABA or glycine had one of three results on the IID function: no effect on suppression from the inhibitory ear, a moderate reduction in the suppression from the inhibitory ear, or an elimination of suppression from the inhibitory ear. Table 1 shows the percent of EI units affected in each of these ways for the GABA blocker and the glycine blocker.

Changes in the IID function are best illustrated by examining changes in the receptive field of the unit. Figure 2 shows the receptive field of an EI unit before and during application of the GABA and glycine blockers. For this unit, both blockers moderately affect the IID function and therefore the receptive field.

Table 1. Effects of blocking GABA and glycine on IID function. Numbers in parentheses indicate the number of neurons.

\section{GABA Blocker \\ (Bicuculline) \\ Glycine Blocker \\ (Strychnine)}

\section{Effect on Suppression from Inhibitory Ear No Effect Moderate Effect Large Effect}
$65.5 \%(55)$
$21.4 \%(18)$
$13.1 \%(11)$
$62.5 \%(25)$
$27.5 \%(11)$
$10.0 \%$

\section{Discussion}

A neuron like the one shown in Figure 2 appears to have its receptive field modified by both GABA and glycine. This type of EI neuron may receive an excitatory input already containing basic EI-like characteristics which are sharpened by GABAergic inputs, presumably from the DnLLs, and by a glycinergic input, presumably from the ipsilateral LSO. A neuron with a receptive field that appears to be unaffected by GABA or glycine is presumably receiving its EI characteristics entirely from excitatory inputs originating from cells where EI characteristics already exist. For some neurons, GABA appears to completely eliminate suppression from the ipsilateral ear. This result indicates that EI characteristics may be created within the IC for some EI neurons through a GABAergic input, presumably from one or both DnLLs. 
This last finding is interesting because there would appear to be no need to create EI neurons in the colliculus since EI neurons already exist at lower levels of the auditory system that project to the IC. It has been assumed for some time that EI neurons in the IC receive their EI characteristics intact through excitatory projections from the SOC. A recent study by Sally and Kelly (9) supports our finding that some EI neurons are created in the IC. These researchers ablated the nuclei of the superior olive in rats but still found EI neurons in the IC. The question of why some EIs are created in the IC while others receive their characteristics from below and still others appear to be a combination of these processes cannot be answered at this time. The neural coding of IIDs for binaural sound localization is obviously very complex. However, determining the underlying circuitry and pharmacology of IID-sensitive EI neurons is bringing us closer to understanding the underlying mechanisms.
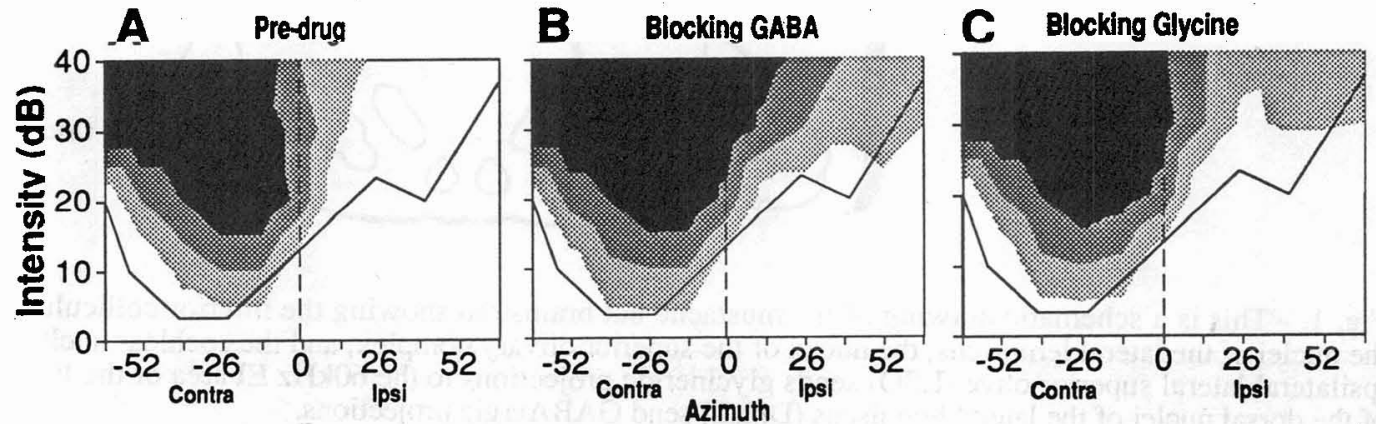

Fig. 2. - These graphs represent the response field of an EI neuron. The $x$-axis indicates azimuthal position in degrees and the y-axis intensity. Progressively darker shades indicate greater responding (black indicates $75-100 \%$ of maximum responding). A: The response field of a typical EI unit. Responsiveness is primarily restricted to the contralateral half of the sound field and sensitivity is greatest at about 26 degrees from the midline, reflecting the directionality of the ear. The line encompassing the response field and extending into the ipsilateral sound field indicates how the boundary of the receptive field would appear if the unit was not suppressed by the ipsilateral ear. B: The GABA blocker, bicuculline, reduces suppression from the ipsilateral ear, extending the receptive field into the ipsilateral sound field. C: The glycine antagonist, strychnine, also extends the receptive field into the ipsilateral sound field, though not as much as the GABA blocker.

\section{References}

/1/ TINBERGEN, N. The Study of Instinct. Clarendon Press, Oxford (1951).

12/ ERULKAR, S.D. Physiological Reviews 52 (1972) 237-360.

13/ LEWIS, D.B. Bioacoustics: A Comparative Approach B. Lewis ed. Academic, London (1983) 233 257.

14/ IRVINE, D.R.F. Progress in Sensory Physiology, Vol. 7 H.Autrum and D. Ottoson eds. SpringerVerlag, Berlin (1986).

15/ POLLAK, G.D., WENSTRUP, J.J., and FUZESSERY, Z. Trends in Neurosci. 9 (1986) 556-561.

/6/ ROSS, L. and POLLAK, G.D. J. Neurosci. 9 (1989) 2819-2834.

III WENSTRUP, J.J., ROSS, L.S., and POLLAK, G.D. J. Neurosci. 6 (1986) 962-973.

/8/ BASBAUM, A. I. and MENETREY, D. J. Comp: Neurol. 261 (1987) 306-318.

19/ SALLY, S. and KELLY, J. B. Brain Research In Press (1991). 